The diameter of a proton is about $10^{-15} \mathrm{~m}$-much greater than that of a quark, but infinitesimal compared with the width of a laboratory proton beam. So when two high energy beams are fired at each other, as in the Intersecting Storage Rings of the European laboratory at CERN, most of the protons do not collide. Those that do nearly always are in glancing collision. The dynamics of such collisions, where only the outer parts of the two protons interact, is complicated. It does not involve the quark structure in a very direct way, and the particles produced in the interaction (mostly pimesons) are swept along more or less in the initial directions of the two colliding beams.

Very rarely, two protons collide head-on and in this case their quark structure may be expected to be involved rather directly. Also, the headon collisions are quite likely to produce particles that emerge with a fast sideways motion relative to the initial beams. Although these 'large-transverse-momentum' events are comparatively very rare, they can readily be identified, and their study has been an important part of the CERN Intersecting Storage Rings research programme during the past four or five years. Some important information has come also from experiments at Fermilab in the United States.

High-energy proton-proton collisions produce a large number of additional particles, making the analysis of the interesting events complicated. So far the analysis cannot be complete because of the limitations of the available particle detectors. However, several of the CERN experiments have recently observed an interesting "jet structure" in the large-transversemomentum events: the sidewaysmoving particles emerge together in more or less the same direction, so as to form a jet of particles. This jet structure has been seen most clearly in three experiments using the CERN Split Field Magnet facility. These experiments were performed by a group from CERN, by a CERNCollège de France-Heidelberg-Karlsruhe collaboration, and by a BritishScandinavian-French collaboration. More recently, a collaboration from Caltech, UCLA, Fermilab, Chicago and Indiana has reported results from a jet study at Fermilab. The total momentum of the two protons that collide is zero, so there are two jets, one emerging to each side of the initial beam direction (see Fig. 1). In addition to the two jets, several particles are produced that have small transverse momentum, as in the ordinary, glancing collisions.

Pairs of jets are known also to be a feature of collisions of high-energy electrons with positrons. In these col-

\section{Transcriptional control of fertility}

from J. R. Saunders

A RECENT paper from Neil Willetts (J. molec. Biol. 112, 141; 1977) provides the first biochemical evidence that the fertility of bacterial plasmids is regulated at the level of transcription. Most of the genes specifying the ability to conjugate in Escherichia coli comprise a single 'transfer operon' which is present on the F factor and F-like plasmids. This operon consists of genes necessary for the production of sex pili, for surface exclusion and for the metabolism of DNA during transfer. It is transcribed in the order $\operatorname{traA}, \operatorname{traL}$, traE, traK, traC, traF, traH, traG, traS, traD (Helmuth \& Achtman Nature 257, 652; 1975). A further gene traJ, has a positive controlling function over the entire operon but lies outside it. In turn the expression of traJ is itself subject to negative control imposed by two further plasmid genes finO and finP acting in concert. F-like plasmids isolated from the wild generally due to this control. $F$ itself is atypical in that it is a naturally occurring fin $\mathrm{O}^{-}$mutant and consequently transfers itself at a high rate. However $F$ will recognise the finO product specified by other F-like plasmids and if present in the same cell such plasmids inhibit $\mathrm{F}$ fertility producing the $\mathrm{Fin}^{+}\left(f^{+}\right)$phenotype.

lisions, the electron and the positron annihilate each other. There is convincing evidence that the energy that they leave behind subsequently materialises as a quark and an antiquark, which apparently do not escape as free particles. Exactly what happens is not understood, but first they "dress" themselves somehow by interacting with each other, so that they then emerge as two jets of ordinary particles. There are also indications that similar jets result from the collisions of high-energy electrons, muons or neutrinos on protons.

Because there is good evidence that

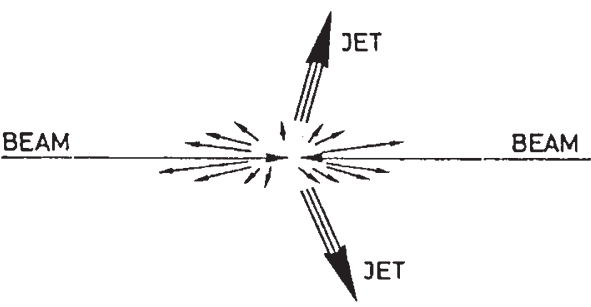

Fig. 1 The two jets produced in large-transverse-momentum collisions together with particles that have small transverse momentum relative to the incoming beams. transfer at a relatively low frequency
Genetic tests have established that the gene products of the transfer operon are absent during FinOP fertility inhibition. Hitherto it was unclear whether the traJ product is necessary for transcription or translation of the operon. Willetts has distinguished between these possibilities by first constructing bacteriophage $\lambda$ transducing phages carrying either traJ or the traL, traE, traK region. He then used DNA from these phages as probes for the synthesis of specific messenger RNA during FinOP transfer inhibition. His hybridisation experiments indicate that whilst mRNA homologous with this 'tra' DNA is present in donor strains carrying $F$ alone it cannot be found during transfer inhibition of suggests that the transcription of traJ is repressed under the latter conditions. In the absence of the traJ product, transcription of the transfer operon itself is either not initiated or possibly terminated prematurely shortly after initiation. It should prove possible now to extend these studies on the expression of sex factors by utilising $\lambda$ tra phages carrying further transfer regions of $F$ and other plasmids.

$J . R$. Saunders is a lecturer in the Department of Microbiology in the University of Liverpool. F by the F-like $R$ plasmid $R 100$. This

these jets originate from quarks, a natural guess is that the same may be true of the jets produced in the largetransverse-momentum proton-proton collisions. In the simplest model for this reaction, a quark from one of the protons scatters a quark from the other through a wide angle. The two quarks then somehow dress themselves, and result in the two jets. The remaining constituents of the two protons are responsible for the production of the longitudinally-moving particles.

There are other models that differ in detail. The experimental and theoretical question of whether the jets seen in proton-proton collisions do have the same character as those in electron-positron annihilation has yet to be answered. But it is already apparent that the large-transversemomentum proton-proton reactions have fairly simple features which, in a way whose details are still to be determined, do reflect the quark structure of the proton. When the dynamics of these reactions are better understood, they will provide a unique way of investigating how quarks scatter on each other at high energies. 